\title{
Does Urban Agglomeration Promote Urban Land Green Use Efficiency? Take the Yangtze River Economic Zone of China as an Example
}

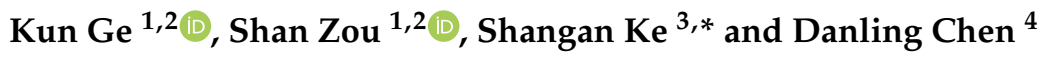 \\ 1 College of City Construction, Jiangxi Normal University, Nanchang 330022, China; akun1900@163.com (K.G.); \\ shan13065153573@163.com (S.Z.) \\ Institute of Real Estate, Jiangxi Normal University, Nanchang 330022, China \\ 3 School of Public Administration, Central China Normal University, Wuhan 430079, China \\ 4 College of Public Administration, Huazhong Agricultural University, Wuhan 430070, China; \\ danlingchen@mail.hzau.edu.cn \\ * Correspondence: keshangan@126.com
}

Citation: Ge, K.; Zou, S.; Ke, S.; Chen, D. Does Urban Agglomeration Promote Urban Land Green Use Efficiency? Take the Yangtze River Economic Zone of China as an Example. Sustainability 2021, 13, 10527. https://doi.org/10.3390/ su131910527

Academic Editor: Pablo Peri

Received: 5 August 2021

Accepted: 18 September 2021

Published: 23 September 2021

Publisher's Note: MDPI stays neutral with regard to jurisdictional claims in published maps and institutional affiliations.

Copyright: (c) 2021 by the authors. Licensee MDPI, Basel, Switzerland. This article is an open access article distributed under the terms and conditions of the Creative Commons Attribution (CC BY) license (https:// creativecommons.org/licenses/by/ $4.0 /)$.

\begin{abstract}
This paper theoretically constructs the logical framework of urban agglomeration affecting urban land green use efficiency (ULGUE) and employs the super efficiency SBM model with undesired outputs to measure ULGUE based on the research object from 107 prefecture-level cities and above in the Yangtze River Economic Zone. Then, this paper uses the difference-in-differences method to test the impact of urban agglomeration on the ULGUE. The results show that: (1) urban agglomeration has a positive regulatory effect on the ULGUE, and this research conclusion is robust; (2) the promotion effect of urban agglomeration on the ULGUE has regional differences, showing the basic pattern of ordinary prefecture-level cities > regional central cities > provincial capital cities and medium cities > big cities > super large and megacities; (3) small and medium-sized cities should be encouraged to further agglomerate while moderately controlling the excessive agglomeration of super-large cities and megacities. Moreover, it is suggested to improve the level of regional agglomeration from the aspects of flexible use of market mechanisms and policy tools, innovative technology applications to achieve the effective integration of urban agglomeration, and ULGUE promotion.
\end{abstract}

Keywords: urban agglomeration; urban land green use efficiency; Yangtze River Economic Zone; difference-in-differences

\section{Introduction}

In recent years, China has successively proposed major strategies, such as new-type urbanization and regional coordinated development, aiming to promote high-quality urban development. Urban agglomeration, as an essential manifestation of these strategies at the spatial level, can affect population flow, the industrial division of labor, and knowledge spillover to change the quality of regional economic development [1] and ultimately reflect the urban land-use efficiency. Theoretically, urban agglomeration strengthens the flow frequency, and integration degree of elements among regions promotes knowledge innovation and technological progress by adjusting resource allocation structure, deepening intercity interaction and cooperation and sharing public infrastructure, thus stimulating new vitality of economic development and improving the efficiency and quality of land use [1,2]. However, in fact, under the existing administrative division management system and fiscal decentralization system, whether it is based on the protection of local industries or the consideration of self-interest maximization in political contests [2], compared with the new-type development model of promoting regional agglomeration to achieve rapid economic development and efficient land use, local governments are more enthusiastic about the "traditional segmentation" development model centered on the jurisdiction [3]. Under this macro background, the impact of urban agglomeration on the efficiency and 
quality of urban land use may not be significant. What cannot be ignored is that in the process of further promoting the development of urban agglomeration, the traditional development model that prefers economic aggregate growth has made the problems of population crowding, industrial isomorphism, environmental pollution, and resource shortage increasingly prominent $[4,5]$. The question of whether urban agglomeration increases or decreases land-use efficiency remains unclear.

At present, the academic research on the impact of urban agglomeration on land-use efficiency is mainly carried out from the following two aspects: one is to take urban agglomeration as a pure macroscopic geographical phenomenon and analyze the land-use level and development efficiency of urban agglomeration, which is the spatial organization form of urban agglomeration [1,4,5]. Related research focuses on the spatial distribution pattern, regional differences, and influencing factors of urban land-use efficiency [6-10]. The second is to focus on the microscopic performance of a particular aspect of urban agglomeration, such as the impact of population agglomeration and industrial agglomeration on urban land-use efficiency. This kind of research focuses on the internal logical relationship between industrial structure [11,12], urbanization level [13-15], inputs of production factors such as capital, labor, innovation [16,17], and urban land-use efficiency under the background of urban agglomeration, which is essentially the evaluation of land-use performance of urban agglomeration. Based on the above research, scholars mostly use econometric analysis tools, such as the DEA model [18], the Durbin model, threshold regression [11], and objective data and spatial views to describe the specific impact of the micro performance of urban agglomeration on urban land-use efficiency. On the whole, these studies provide a knowledge overview and theoretical support for grasping the relationship between urban agglomeration and urban land-use efficiency. In particular, some studies take the three waste pollutant emissions caused by urban land use as undesired outputs and incorporate this kind of output into the measurement system of urban land-use efficiency [19-23], to implement the concept of green development into the process of urban land use and realize the green use and production of urban land. Regrettably, the above results primarily focus on exploring urban land-use efficiency under urban agglomeration from the perspective of "environment for growth" but ignore the "low carbon" attribute of green development [20]. Moreover, the existing research paradigm of "urban agglomeration-urban land-use efficiency" does not systematically reveal the mechanism of urban agglomeration on urban land-use efficiency. Therefore, it is impossible to deduce the empirical context of urban agglomeration affecting urban land-use efficiency from theoretical logic, and, then, the relevant regional land-use policies are suspended.

Given this, starting from the concept of green development, this paper attempts to construct a theoretical framework of urban agglomeration affecting urban land green use efficiency (ULGUE). Taking 107 prefecture-level cities and above in the Yangtze River Economic Zone as the research object, considering multiobjective constraints such as energy saving, emission reduction, and low carbon, the super efficiency SBM model is used to analyze the basic level of ULGUE, and the difference-in-differences method is used to empirically test the driving effect of urban agglomeration on ULGUE. In addition, this paper further examines the moderating effect of different levels and scales of cities on the promotion of ULGUE under the background of urban agglomeration development, to provide theoretical and empirical support for exploring the differentiated promotion path of regional ULGUE (Figure 1). 


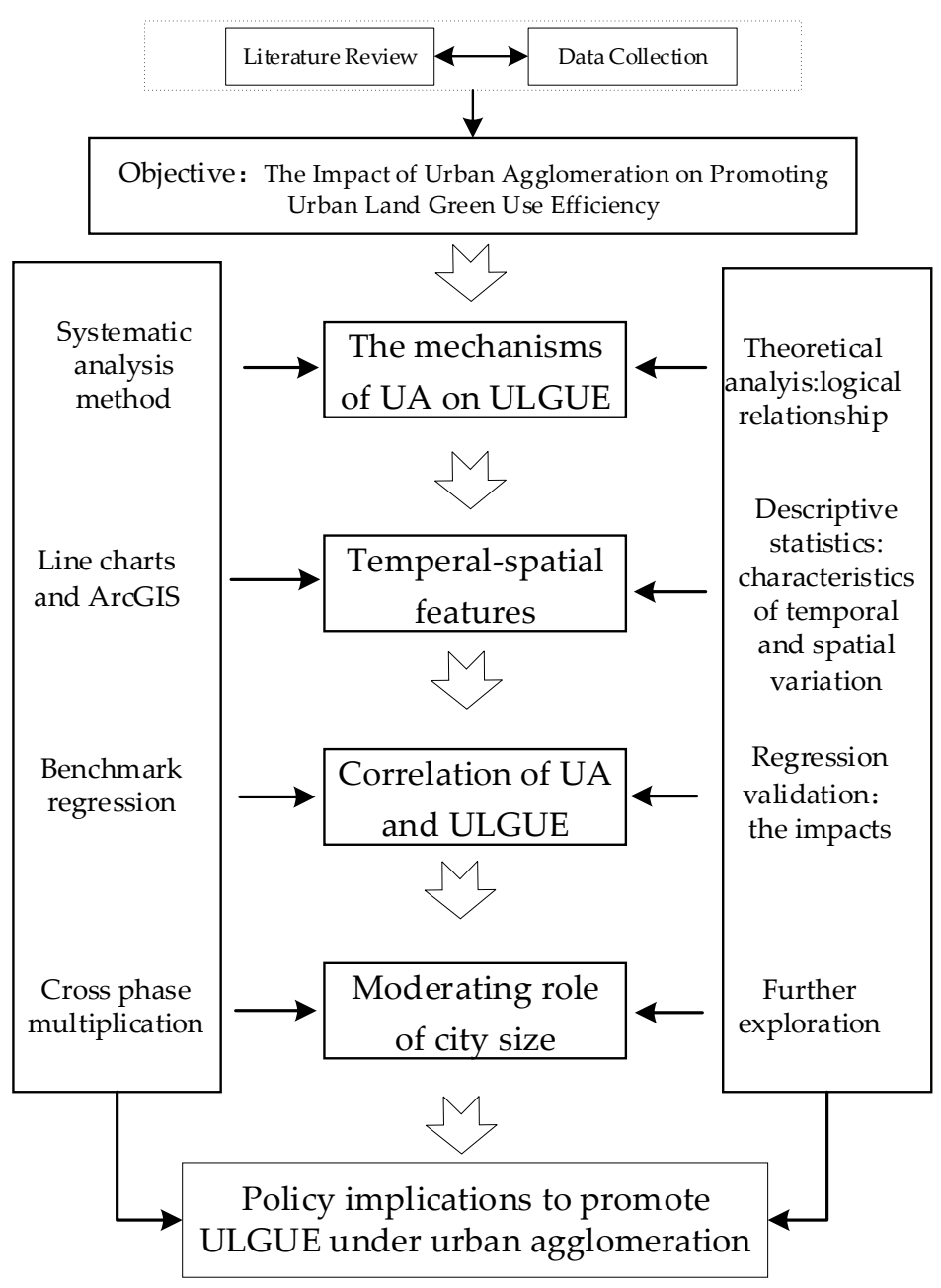

Figure 1. The flow chart of this paper.

\section{Theoretical Framework: Influence Mechanism of Urban Agglomeration on ULGUE}

Under the integration development of urbanization and industrialization, cities with different scales, functions, and administrative levels have different degrees of attraction to factors such as labor, capital, and technology, which makes the urban agglomeration development present a spatial distribution pattern of "center-periphery". In this pattern, the changes of flow direction, configuration structure, and coordination degree of each factor directly or indirectly affect regional economic activities, and then affect ULGUE under the interaction. Specifically, urban agglomeration mainly affects ULGUE through polarization effect, trickle-down effect, and constraint effect (Figure 2). 


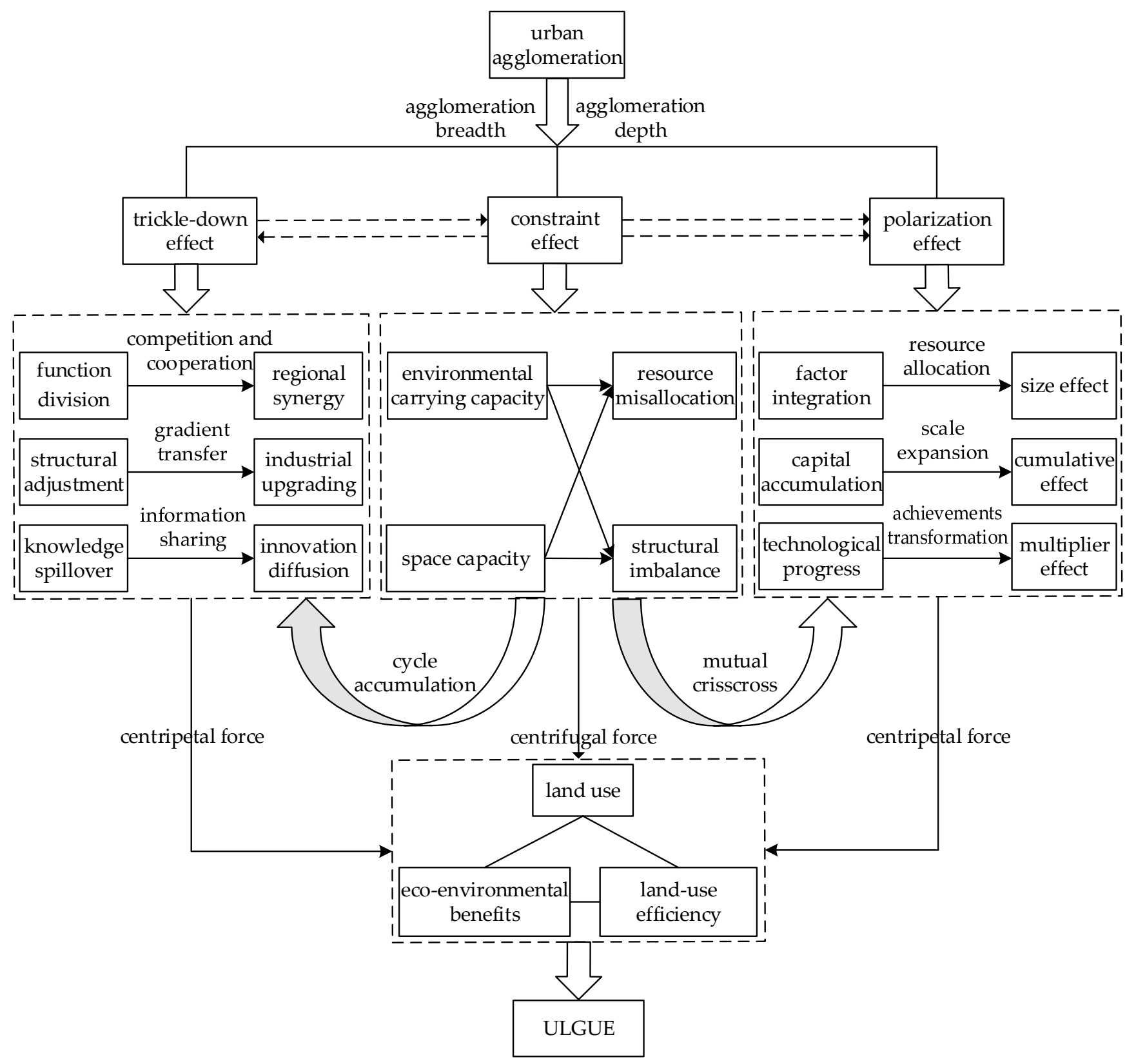

Figure 2. The influence mechanism of urban agglomeration on ULGUE.

\subsection{Polarization Effect}

There is a strong siphon effect in central cities in urban agglomeration areas, which leads to the flow of labor force, capital, technology, and other elements from noncenter cities to the central city [24]. In the short term, the disparity of ULGUE level between cities can be increased, but in the long term, the center city can revitalize various elements in the process of agglomeration, produce scale effect, investment multiplier effect, and energy level diffusion effect, drive the development of peripheral cities, and then promote the joint development of ULGUE [1-3]. Specifically, on the one hand, factor integration and capital accumulation can help central cities reduce labor costs, enrich intermediate product markets, and share infrastructure, and then optimize factor allocation efficiency, and improve regional economic outputs and urban land-use efficiency $[25,26]$. On the other hand, population agglomeration and spatial expansion increase the demand for consumer products and public services, and further promote the industrial-scale expansion of central cities. Under the effect of market supply and demand alternate strengthening, the 
industries in central cities are developing in the direction of rationalization and advanced structure, to enhance the impetus of urban economic development, improve the income per unit of land, and promote the improvement of urban land-use efficiency. At the same time, knowledge subjects with diversified and discrete characteristics can be complementary and integrated into the central city [25], to achieve technological progress in scientific and technological innovation activities and knowledge sharing and collaboration, and promote the transformation of economic development from factor-driven to innovation-driven, and ultimately improve the level of ULGUE.

\subsection{Trickle-Down Effect}

The continuous expansion of urban agglomeration breadth strengthens the trickling effect of central cities on peripheral cities [24], and thus promotes different cities to form a development community with complementary functions and regional synergy in the process of competition and cooperation, industrial transfer, and knowledge spillover. Under the central-peripheral spatial development pattern, some elements of central cities are selectively transferred to the potential market and the peripheral cities with lower production, and the energy level transfer mechanism between cities is gradually optimized, thus promoting the coordinated development of economy and land use in urban agglomeration areas [27]. Specifically, on the one hand, the dynamic evolution of factor agglomeration-diffusion and the interaction of competition and cooperation between cities have promoted the division of labor in agglomeration areas from interindustry or intraindustry division to specialization and diversification of spatial functions based on the value chain [28], thus promoting the synergistic improvement of ULGUE. On the other hand, peripheral cities accelerate economic development by undertaking the industrial transfer of central cities and optimizing the internal industrial structure, and gradually narrow the gap with the central city in the process of industrial structure upgrading [29]. In addition, the knowledge spillover and technology diffusion effect formed in this process gradually penetrates different production sectors to play a role, and the resulting "urban agglomeration integration" significantly promotes the changes in the economic and social fundamentals of urban agglomeration areas, and inevitably impacts ULGUE.

\subsection{Constraint Effect}

The dual constraints of resources and the environment in the process of urban agglomeration are essential factors to reduce ULGUE. Due to the limited space capacity and environmental carrying capacity, the excessive accumulation of population, industry, and capital and the resulting resource mismatch and structural imbalance causes external uneconomic problems, such as shortage of water and soil resources, deterioration of the ecological environment, and traffic congestion [4,30], which undermine the driving force of urban green development and thus affect the level of ULGUE. With the improvement of urban agglomeration level and the expansion of urban agglomeration scale, the constraint effect of the deterioration of the abovementioned resource and environmental conditions on ULGUE in agglomeration areas are further manifested.

To sum up, whether urban agglomeration is beneficial to improve ULGUE is the result of the mutual restraint of polarization effect, trickle-down effect, and restraint effect. However, the influencing mechanism of ULGUE changes is complex, and the existence of the endogeneity problem makes it difficult to clarify the impact of urban agglomeration on ULGUE. The lack of critical variables is a well-known endogeneity cause, and more and more researchers have realized that it is not easy to eliminate the endogeneity problem in empirical studies by relying only on the measurement technology itself [31]. Fortunately, the natural experiment of urban agglomeration construction in China provides a unique opportunity to study the relationship between urban agglomeration and ULGUE. 


\section{Overview of the Study Area}

The Yangtze River Economic Zone, with the Yangtze River as the link, runs through nine provinces and two cities in Shanghai, Jiangsu, Zhejiang, Anhui, Jiangxi, Hubei, Hunan, Chongqing, Sichuan, Yunnan, and Guizhou, including three national-level cross-regional urban agglomerations: the Yangtze River Delta urban agglomeration, urban agglomeration in the middle reaches of the Yangtze River, and the Chengdu-Chongqing urban agglomeration (Figure 3). As a significant economic growth pole of the Yangtze River Economic Zone, in 2019, the three national-level urban agglomerations gathered about $30 \%$ of the country's population with about $8 \%$ of the land area, and created about $40 \%$ of the gross domestic product, which is a vital population, resources, and industrial agglomeration area in China. However, as the level of urban agglomeration continues to deepen, in the process of cluster development of the three national urban agglomerations, the problems such as rising labor costs, tightening resource and environmental constraints, and insufficient regional development motivation have become increasingly prominent [32], affecting the further improvement of ULGUE. With the development of urban agglomeration presenting the development dilemma of high resource consumption, high pollution emission, and high-carbon emission coexisting in urban land use, does urban agglomeration improve ULGUE? The answer to this question is of great practical significance for testing the effectiveness of urban agglomeration development strategy and the reasonable planning and layout of the main body of urban agglomerations in the future.

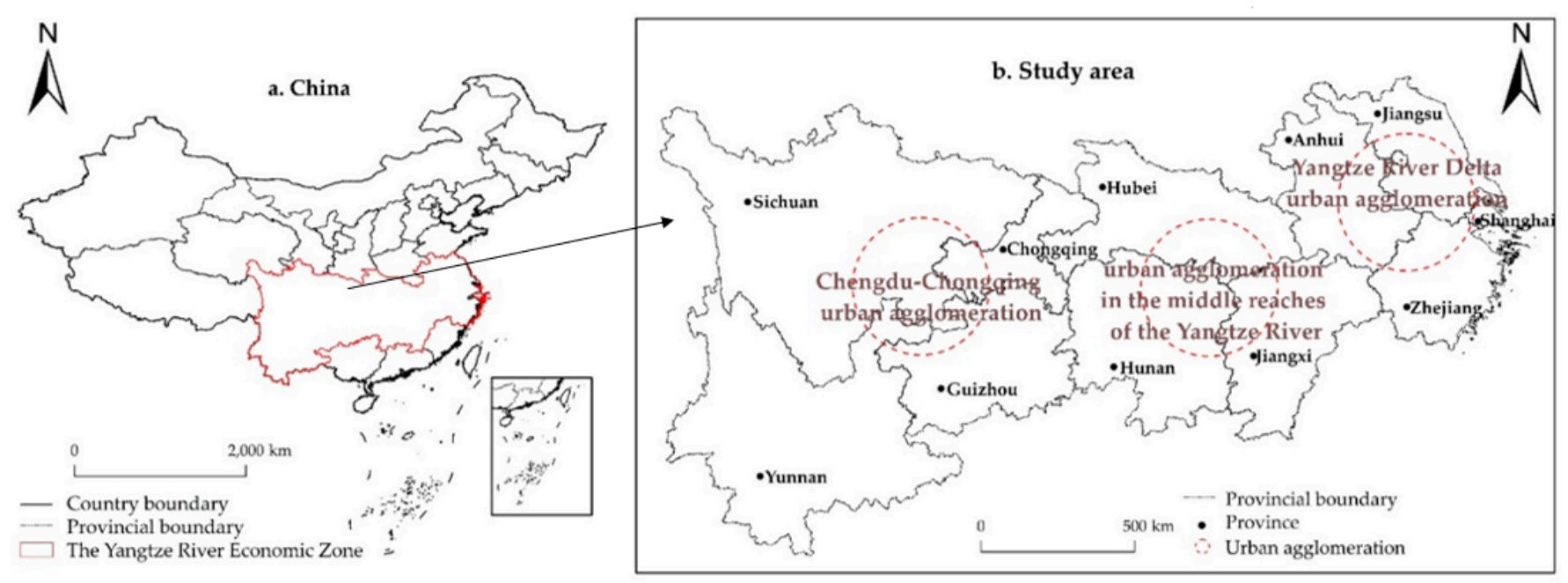

Figure 3. The spatial scope of the Yangtze River Economic Zone in China.

\section{Model Setting and Variable Measurement}

\subsection{Model Setting}

\subsubsection{The Super Efficiency SBM Model for Measuring ULGUE}

The super efficiency slack-based model (SBM) is a data envelopment analysis method that contains slack variables and undesired outputs proposed by Tone [19]. Compared with the traditional data envelopment analysis, this model can solve the problem of inputoutput relaxation on the one hand; on the other hand, it can also solve the problem of efficiency analysis under the existence of unexpected output. Therefore, it is widely used in urban environmental efficiency [33], urban land-use efficiency [34], and industrial green development efficiency [35]. Considering the loss of resources, environment, and ecological efficiency in the urban land green use, this paper adopts the super efficiency SBM model 
based on undesired outputs to measure the ULGUE level of cities in the Yangtze River Economic Zone. The basic model is formulated as follows:

$$
\begin{gathered}
\min \rho=\frac{\frac{1}{m} \sum_{i=1}^{m}\left(\bar{x} / x_{i k}\right)}{\frac{1}{r_{1}+r_{2}}\left(\sum_{s=1}^{r_{1}} \overline{y^{d}} / y_{s k}^{d}+\sum_{q=1}^{r_{2}} \overline{y^{u}} / y_{q k}^{u}\right.} \\
\left\{\begin{array}{cc}
\bar{x} \geq \sum_{j=1, \neq k}^{n} x_{i j} \lambda_{j} & i=1,2 \cdots, m \\
\frac{y^{d} \leq \sum_{j=1, \neq k}^{n} y_{s j}^{d} \lambda j}{y^{d} \geq \sum_{j=1, \neq k}^{n} y_{q j}^{u} \lambda j} & q=1,2 \cdots, r_{1} \\
\frac{\lambda_{j} \geq 0}{\bar{x} \geq x_{k}} & j=1,2 \cdots, r_{2} \\
\frac{y^{d} \leq y_{d}^{k}}{y^{u} \geq y_{k}^{u}} & q=1,2 \cdots m \\
u=1,2 \cdots, r_{2}
\end{array}\right\}
\end{gathered}
$$

In Equations (1) and (2), $n$ indicates the number of decision-making units; $m, r_{1}$, and $r_{2}$, respectively, represent the input, expected outputs, and undesired outputs of the decision-making unit; $x, y^{d}$, and $y^{u}$ are the number of elements in the corresponding input matrix, expected matrix, and undesired matrix; $\rho$ is the value of the urban land green use efficiency.

\subsubsection{The Difference-in-Differences Method for Measuring the Effect of Urban Agglomeration on ULGUE}

The difference-in-differences method is a quantitative analysis method for estimating causal effects. Compared with the single-difference method and the traditional econometric model, in the case of controlling the difference between the experimental group and the control group in advance, the difference-in-differences method can effectively separate the net impact of policy or project implementation by constructing natural experiments. So, its calculation results are more accurate and reliable [36] and can be an ideal model for testing the impact of urban agglomeration on ULGUE. According to the difference-indifferences model, 69 prefecture-level cities and above included in the Yangtze River Delta urban agglomeration, urban agglomeration in the middle reaches of the Yangtze River, and Chengdu-Chongqing urban agglomeration in the Yangtze River Economic Zone are used as agglomeration areas (experimental group), and another 38 prefecture-level cities are used as nonaggregated areas (control group). The paper regards the establishment of the agglomeration area (national-level urban agglomeration) as a quasi-natural event, applying the difference-in-differences method to identify the net impact of urban agglomeration on ULGUE. As each city joins the city agglomeration at different time nodes, referring to Liu Ruiming's and Yuan Jianguo's handling methods [36,37], this paper uses a twoway fixed-effect model to achieve the difference-in-difference effect. The model is built as follows:

$$
U L G U E_{i t}=\beta_{0}+\beta_{1} U A_{i t}+\gamma X_{i t}+v_{i}+\mu_{t}+\varepsilon_{i t}
$$

In Equation (3), $i$ represents the urban individual; $t$ represents the year; the explained variable $U L G U E_{i t}$ represents the urban land green use efficiency of the $i$ area in the year $t$; the core explanatory variable $U A_{i t}$ is a dummy variable reflecting whether the $i$ area has joined the urban agglomeration in the year $t$; before joining the urban agglomeration, $U A_{i t}=0$; after joining, $U A_{i t}=1 ; \beta_{1}$ represents the net benefit of urban agglomeration to urban land green use efficiency; $X_{i t}$ is the set of control variables, and $\gamma$ is the corresponding control variable coefficient; $v_{i}$ and $\mu_{t}$, respectively, represent individual fixed effects and time-fixed effects; $\varepsilon_{i t}$ is a disturbance term that varies with individuals and time. 


\subsection{Variable Description}

\subsubsection{Explained Variable: Urban Land Green Use Efficiency (ULGUE)}

ULGUE is an important indicator that measures the intensity and degree of adaptation of land use inputs and outputs under multiple goals such as the optimization of the scale of resource utilization, the minimization of environmental pressure, and the synergy ascension of socioeconomic-ecological outputs under the concept of green development [38,39]. Unlike the previous concept of urban land use that only focuses on solving a certain economic or environmental problem, it emphasizes the ability and potential of urban land use to reach the optimal level under the triple constraints of energy saving, emission reduction, and low carbon. Therefore, ULGUE needs to further consider the dynamics of green and sustainable development on urban land use based on calculating the allocation scale of conventional land, manpower, capital, and the benefits of socioeconomic-ecological output. Therefore, it is necessary to include undesired indicators such as energy consumption, environmental pollution, and carbon emissions at the same time.

For the selection of specific indicators, this paper refers to the ULGUE measurement ideas of scholars such as Liang Liutao [38], Wang Deqi [39], and Lu Xinhai [40] (Table 1). They construct the evaluation system from the perspective of input index and production place index (expected output and unexpected output), select input index mainly from the dimension of production factors and energy, screen expected index from the angle of social economic ecological impact, and consider expected output from the angle of industrial waste. (1) In addition to considering the input quantity of land, capital, and labor force, the input indicators also try to consider the dynamic change of energy input scale in time. This paper selects the built-up area, fixed asset investment per land, tertiary industry employees per land, and electricity consumption per unit of GDP to characterize them. (2) The expected outputs indicators mainly consider the development of economic, social, and green ecological aspects brought about by urban land use. The total GDP can be used to characterize the economic output level of the land to a certain extent. To enhance the comparability of the data, the year 2003 is the base period, using the regional GDP index to convert nominal GDP into comparable actual GDP; the social development index can reflect the level of social development in the region and the improvement of people's livelihood. This paper uses the entropy weight method to calculate the social development index by selecting population growth rate, the proportion of the population with a college degree or above in the total population, and the number of hospital beds per 10,000 people; expanding the green area of built-up areas is an eco-environment-friendly urban land-use method. This paper selects the green area coverage of the built-up areas as a measure of the ecological quality of urban land use. (3) In the specific selection of undesired outputs indicators, considering that water pollution is an important cause of negative external environment impacts in the Yangtze River Economic Zone, the emission of industrial wastewater is included in the emission reduction index, as well as the emission of solid waste and industrial $\mathrm{SO}_{2}$, and the entropy weight method is used to synthesize the three into a comprehensive pollutant emission index to reflect the environmental pollution status; in addition to the conventional "three wastes" pollutants, the urban land-use process also releases a certain amount of $\mathrm{CO}_{2}$. So, in the sustainable dimension, energy carbon emissions indicators have been added to the undesired outputs, including carbon emissions from the consumption of raw coal, coke, gasoline, kerosene, diesel, fuel oil, liquefied petroleum gas, natural gas, cleaned coal, other coal washing, and coke oven gas consumption. 
Table 1. The ULGUE measurement.

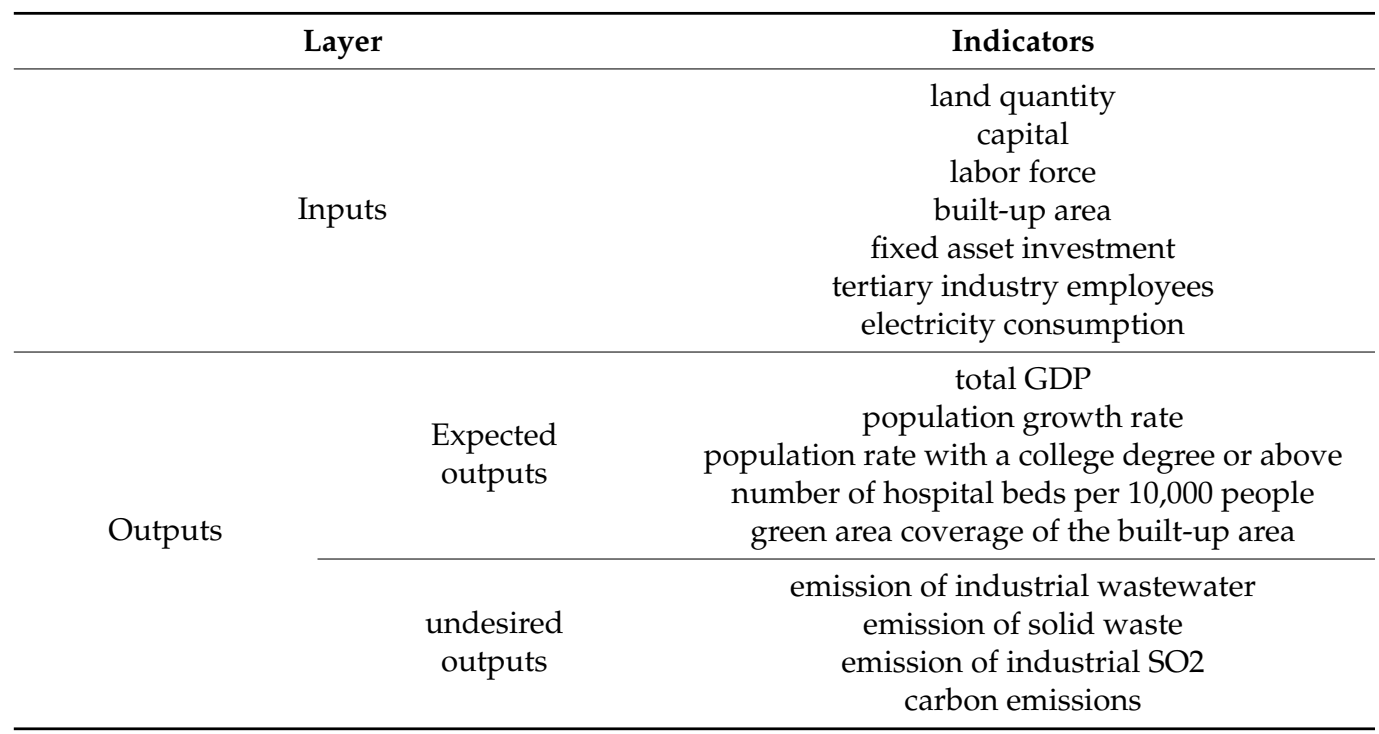

\subsubsection{Core Explanatory Variable: Urban Agglomeration (UA)}

This paper focuses on exploring whether the development of urban agglomeration areas can improve ULGUE, setting urban agglomeration as a dummy variable and recording the year $t$ that each city in the Yangtze River Economic Zone joins the urban agglomeration as 1 , otherwise it is recorded as 0 . The regression relationship between whether to join a national-level urban agglomeration and ULGUE is used to test the impact of urban agglomeration on ULGUE

\subsubsection{Control Variables}

Concerning the relevant research of Hu Bixia and other scholars [34,39,41], the following indicators are selected as the control variables that affect ULGUE: (1) the macro level mainly selects the level of government intervention GI (local fiscal expenditure/GDP) and the level of infrastructure investment INFR (public infrastructure financial investment/GDP); (2) the meso level mainly considers the advanced industrial structure IS (output of the tertiary industry/output of the secondary industry) and the rationalization of the industrial structure RS (calculate the Theil index to measure); (3) the micro level mainly considers technology innovation level TI (technology research and development investment/GDP).

\subsection{Data Source and Description}

As an island, Zhoushan city in Zhejiang Province was not included in the research scope. In addition, the original Chaohu city was revoked and placed under the management of Hefei city in 2011. Therefore, this paper finally selected 107 prefecture-level cities and above in the Yangtze River Economic Zone as the research sample: among them were 31 urban samples in the upstream region, 36 urban samples in the midstream region, and 40 urban samples in the downstream area. The main sources of social and economic data are the China City Statistical Yearbook and the provincial statistical yearbook from 2004 to 2020, and a small number of missing values are supplemented by the linear interpolation method. The descriptive statistics of the variables involved in the paper are shown in Table 2. 
Table 2. Descriptive analysis of the variables.

\begin{tabular}{cccccccc}
\hline Type & Variable & Symbol & Obs. & Max. & Min. & Mean. & S.D. \\
\hline explanatory variable & urban agglomeration & UA & 1819 & 1 & 0 & \\
\hline explained variable & urban land green use efficiency & ULGUE & 1819 & 1.7146 & 0.1672 & 0.6171 & 0.2865 \\
\hline & level of government intervention & GI & 1819 & 64.8833 & 2.4790 & 14.8268 & 7.1067 \\
& level of infrastructure investment & INFR & 1819 & 67.2118 & 0.3764 & 7.3605 & 6.5291 \\
control variable & advanced industrial structure & IS & 1819 & 9.0569 & 0.2393 & 0.9769 & 0.5759 \\
& rationalization of industrial structure & RS & 1819 & 1.1871 & 0.0000 & 0.2089 & 0.2034 \\
& technology innovation level & TI & 1819 & 2.9825 & 0.0008 & 0.2555 & 0.2940 \\
\hline
\end{tabular}

\section{Empirical Analysis}

5.1. The Temporal and Spatial Evolution of ULGUE under the Background of Urban Agglomeration

Based on the basic principle of the super-efficiency SBM model, the ULGUE of 107 cities along the Yangtze River Economic Zone from 2003 to 2019 was calculated by MaxDEA software, and the ULGUE was analyzed from two aspects of temporal evolution and spatial pattern.

\subsubsection{Timing Evolution}

Figure 4 shows the time series of ULGUE changes in the Yangtze River Economic Zone. On the whole, although the ULGUE level of the whole Yangtze River Economic Zone, agglomeration areas, and nonagglomeration areas have shown the same trend of wavelike rises, the ULGUE increase rate in urban agglomeration areas is generally higher than that in nonagglomeration areas. Specifically, the average annual growth rate of ULGUE during the study period was the highest in urban agglomeration areas $(2.72 \%)$, followed by the whole Yangtze River Economic Zone (2.28\%), and the lowest in nonagglomeration regions (1.52\%). In particular, this is thanks to the continuous improvement of the green development planning system for urban agglomeration in the Yangtze River Economic Zone, such as the promulgation of the Ecology and Environmental Protection Plan for the Yangtze River Economic Zone. From 2017 to the present, the average value of ULGUE from high to low has been the urban agglomeration area (0.7744), the whole Yangtze River Economic Zone (0.7591), nonaggregated areas (0.7313). The fact that ULGUE in agglomeration areas is gradually higher than that in nonagglomeration areas reflects that urban agglomeration and ULGUE have a certain relationship. However, many factors affect ULGUE, and it cannot be inferred from this that the improvement of ULGUE is caused by urban agglomeration. The relationship between the two is empirically tested below.

\subsubsection{Spatial Pattern}

Figure 5 shows the spatial pattern of ULGUE in the Yangtze River Economic Zone using ArcGIS 10.2 software. Overall, the ULGUE level along the Yangtze River Economic Zone shows apparent regional differentiation and high-value local agglomeration characteristics. Specifically, (1) from the perspective of intercity differences, the coefficient of variation of ULGUE between cities rose from 0.3522 in 2003 to 0.4717 in 2019, indicating that the difference in ULGUE between cities has expanded. (2) From the perspective of spatial agglomeration, the high values of ULGUE mainly appear in a few cities in Sichuan and Yunnan at the beginning of the study. Later, the urban agglomeration areas represented by the Yangtze River Delta urban agglomeration and urban agglomeration in the middle reaches of the Yangtze River gradually show a synergistic rise in ULGUE. By the end of the study, the high values of ULGUE are mainly distributed in the urban agglomerations in the middle reaches of the Yangtze River and the Yangtze River Delta urban agglomerations located in the lower reaches of the Yangtze River. (3) From the perspective of city types, after considering the undesired outputs, some prefecture-level cities in Jiangsu, Anhui, and Zhejiang take the lead in the average ULGUE during the study period, while among 
the municipalities and provincial capitals, the average values of Shanghai, Nanjing, and Hangzhou maintain a relatively high level, but do not show apparent advantages. The above spatial pattern shows that there is a certain degree of coordination between the improvement of ULGUE and the development level of urban agglomeration, and the ULGUE of different cities in the context of urban agglomeration may be affected by factors such as city scale, administrative level, and geographic location.

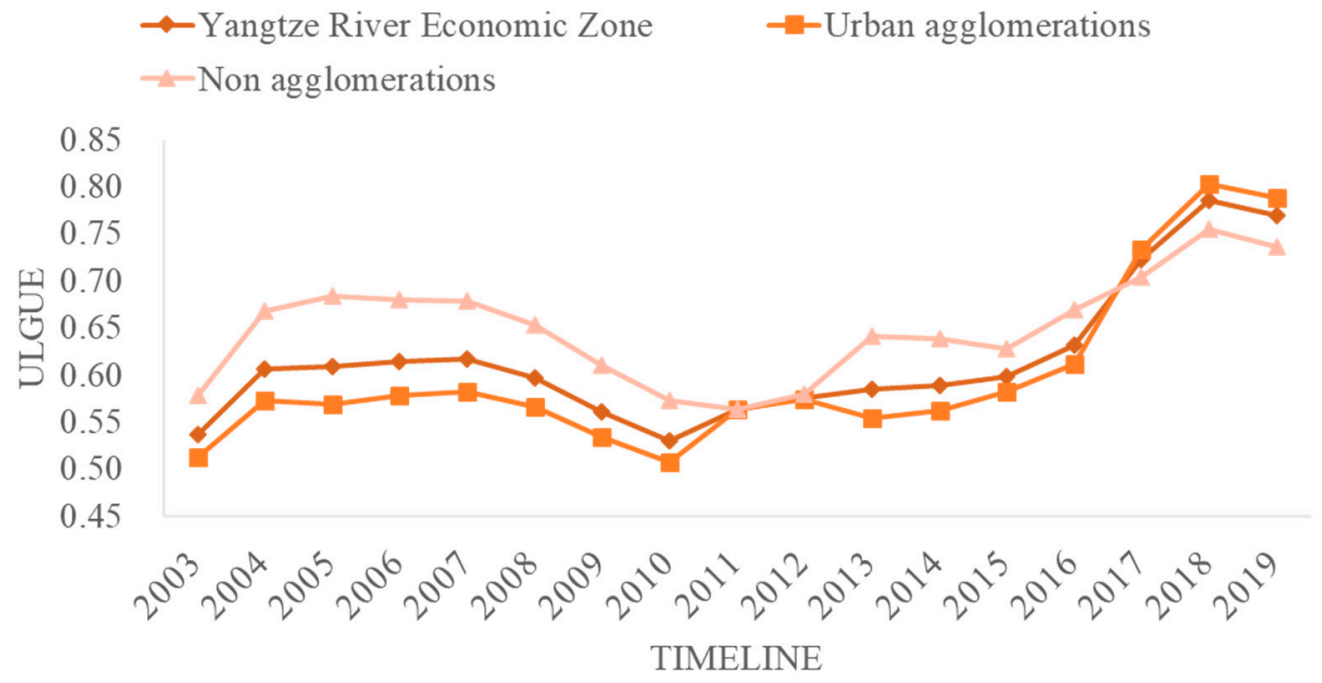

Figure 4. Temporal variation of ULGUE in Yangtze River Economic Zone.

\subsection{Benchmark Regression Results}

Stata16.0 software was used to focus on the net impact of urban agglomeration on ULGUE for regression tests. First, to ensure the validity of the model, multicollinearity was diagnosed for explanatory variables and control variables. The variance inflation factor (VIF) of each variable is within 1.31, and the average VIF is 1.19, indicating no severe multicollinearity problems between the variables.

Secondly, the two-way fixed-effects model of panel data is used to control the region and time simultaneously to achieve the difference in differences effect. To ensure the credibility of the model, the random-effects model is introduced for comparison. It can be seen from Table 3 that in the regression results of the fixed-effects model and the random-effects model with or without control variables, as the dummy variable, urban agglomeration (UA) is significantly positive at the $1 \%$ confidence level, confirming that urban agglomeration has a positive effect on ULGUE. Specifically, under the premise that other variables remain unchanged, compared with cities that have not joined the urban agglomeration, the urban agglomerations formed by joining the urban agglomeration has an average lift of 0.0345 for ULGUE; while considering the control variables in this paper, the average contribution of urban agglomeration increased to 0.0480 . This conclusion shows that the positive effect of the polarization-trickle-down impact of the urban agglomeration area in the Yangtze River Economic Zone during the study period is higher than the negative effect caused by the congestion constraint. It reflects that accelerating the cooperation and interaction of material flow, information flow, capital flow, and other factor flows among cities helps to form the agglomeration effect of group heating and complementary advantages [42], and then improves the ULGUE level. 


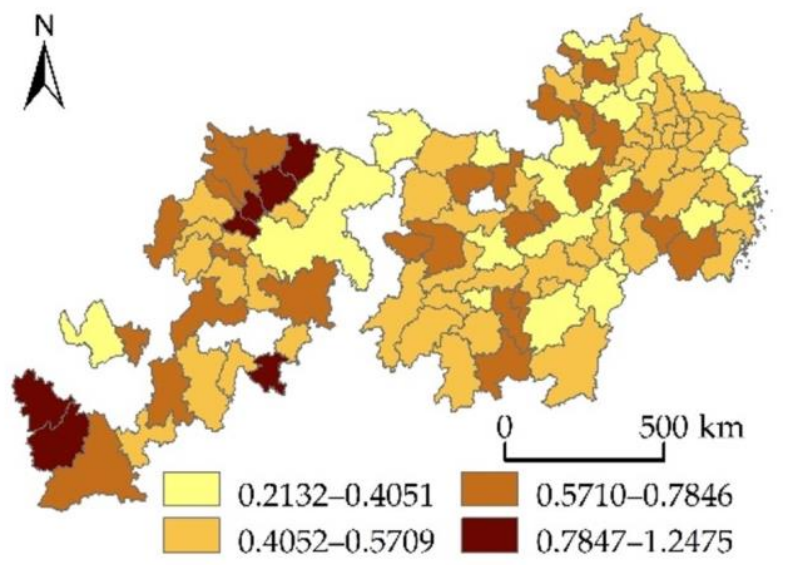

(a)

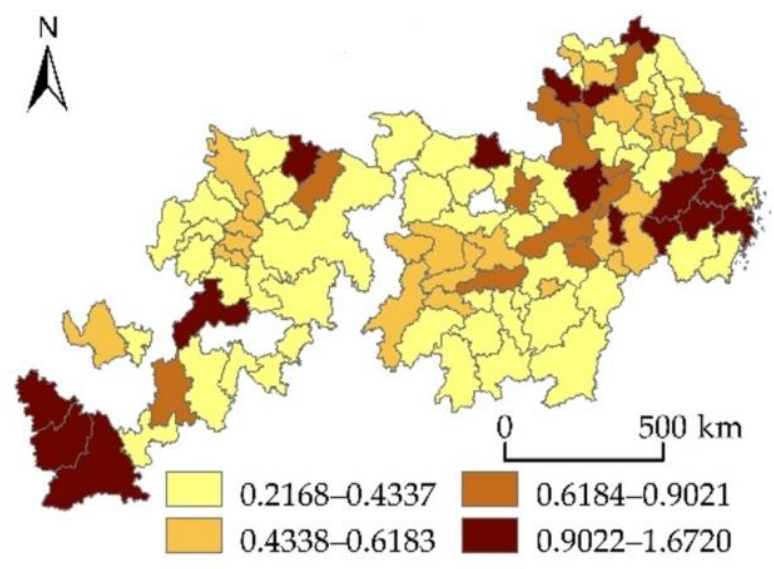

(c)

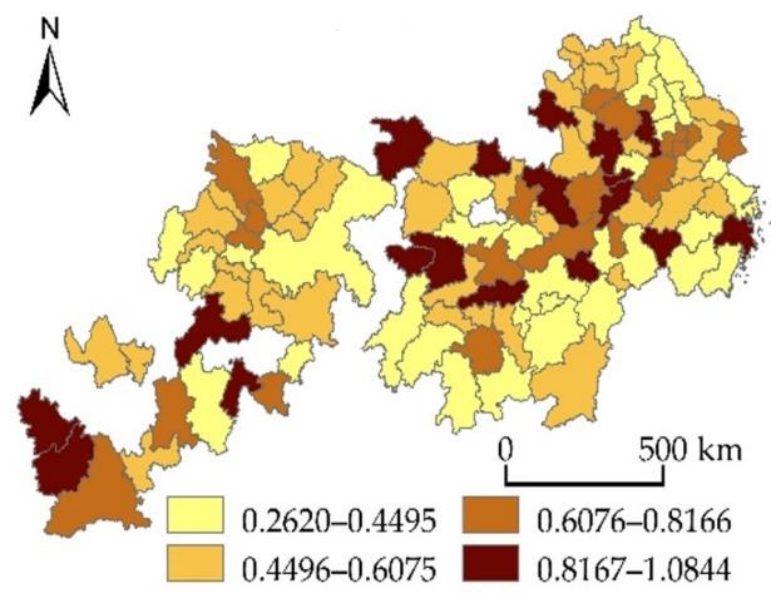

(b)

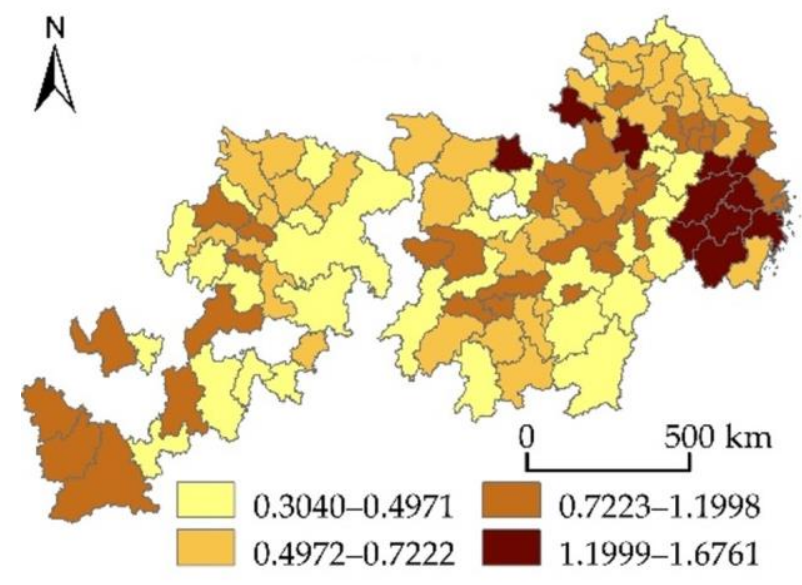

(d)

Figure 5. The spatial distribution of ULGUE in the Yangtze River Economic Zone. (a) ULGUE in 2003; (b) ULGUE in 2008; (c) ULGUE in 2014; (d) ULGUE in 2019.

Finally, column (2) is used as the benchmark regression result to further analyze the influence of each control variable on ULGUE. Among them, the degree of government intervention (GI) has a significant negative impact on ULGUE at the level of $1 \%$. Analysis of the reasons is that in an environment where the market allocates resources, it is similar to the curse effect of political resources on ULGUE [36]; the increase in the degree of government intervention does not necessarily promote the rise of regional ULGUE, in turn, it may hinder the high-quality development of land green use. The impact of industrial structure advancement (IS) and industrial structure rationalization (RS) on ULGUE is significantly positive, indicating that the effects of land green use transformation are represented by the increase in the proportion of the output of the tertiary industry that has continued to show. In addition, there is no evidence to directly indicate that the level of infrastructure investment (INFR) significantly affects the ULGUE level of agglomeration areas. The possible reason is that under the realistic background that the infrastructure is gradually improved and the intercity links are still highly dependent on existing administrative divisions, the regional differences in the impact of the above variables on ULGUE are not obvious. 
Table 3. Impacts of UA on ULGUE.

\begin{tabular}{|c|c|c|c|}
\hline \multirow{2}{*}{ Variable } & \multicolumn{2}{|c|}{ Fixed-Effects Model } & \multirow{2}{*}{$\begin{array}{c}\text { Random-Effects Model } \\
\text { (3) }\end{array}$} \\
\hline & (1) & (2) & \\
\hline UA & $\begin{array}{c}0.0345^{* * *} \\
(0.000)\end{array}$ & $\begin{array}{c}0.0480 * * \\
(0.012)\end{array}$ & $\begin{array}{c}0.0328^{* * *} \\
(0.006)\end{array}$ \\
\hline GI & & $\begin{array}{c}-0.0552^{* * *} \\
(0.001)\end{array}$ & $\begin{array}{c}-0.0623^{* * *} \\
(0.002)\end{array}$ \\
\hline INFR & & $\begin{array}{c}-0.0010 \\
(0.640)\end{array}$ & $\begin{array}{c}-0.0011 \\
(0.304)\end{array}$ \\
\hline IS & & $\begin{array}{l}0.2311^{* * *} \\
(0.000)\end{array}$ & $\begin{array}{l}0.1367^{* * * *} \\
(0.000)\end{array}$ \\
\hline RS & & $\begin{array}{l}0.1401 * \\
(0.053)\end{array}$ & $\begin{array}{l}0.0729^{* *} \\
(0.021)\end{array}$ \\
\hline TI & & $\begin{array}{c}0.1795^{* * *} \\
(0.000)\end{array}$ & $\begin{array}{c}0.2107^{* * *} \\
(0.000)\end{array}$ \\
\hline _cons & $\begin{array}{c}0.4000^{* * *} \\
(0.000)\end{array}$ & $\begin{array}{c}0.4838^{* * *} \\
(0.000)\end{array}$ & $\begin{array}{c}0.5052^{* * *} \\
(0.000)\end{array}$ \\
\hline region-fixed effect & YES & YES & $\mathrm{NO}$ \\
\hline time-fixed effect & YES & YES & NO \\
\hline Obs. & 1819 & 1819 & 1819 \\
\hline R-square & 0.2878 & 0.2952 & 0.1285 \\
\hline
\end{tabular}

Note: ${ }^{*}, *$, and ${ }^{* * *}$ represent significance at $10 \%, 5 \%$, and $1 \%$ statistical level respectively; $p$ value is in parentheses.

\subsection{Robustness Test}

To ensure the rationality of the selected regression model and the accuracy of the estimated results, this paper also conducts the following three robustness tests based on the benchmark regression: (1) introduction of the explained variables with a lag of one period. The ULGUE of each city not only be affected by factors such as industrial structure and technological innovation but may also be affected by the previous ULGUE level. Generally speaking, cities with better ULGUE in the last period tend to maintain a higher level of land green use efficiency in the subsequent period. If the influence of this development path dependence is ignored and included in the random disturbance term, it is easy to cause the endogeneity problem of the model, which leads to biased estimation coefficients, and it is difficult to measure the real impact of urban agglomeration on ULGUE. Therefore, in this paper, the explained variable with a lag of one period is included in the regression model as an instrumental variable for further testing. The regression results are shown in Table 4. After adding the explained variable with a lag of one period, urban agglomeration still has a significant positive impact on ULGUE. (2) Replacement of the estimation method. The ordinary least squares method is used to estimate the relationship between urban agglomeration and ULGUE again. The regression results are shown in the third column of Table 4. The significance of the results is slightly lower than the above results, but it still proves that urban agglomeration does help improve the ULGUE level. (3) Replacement of the control variable. The number of college students owned by 10,000 people is used as a proxy variable of human capital to replace technological innovation as a new control variable for regression testing. The results in the fourth column of Table 3 show that urban agglomeration promotes the improvement of ULGUE. The signs of the three types of robustness test results are consistent with the benchmark regression, indicating that the benchmark estimation results are reliable. 
Table 4. Robustness test results.

\begin{tabular}{|c|c|c|c|}
\hline Variable & $\begin{array}{c}\text { The Explained Variable of } \\
\text { Lag Phase }\end{array}$ & Ordinary OLS Return & $\begin{array}{l}\text { Replace Control } \\
\text { Variables }\end{array}$ \\
\hline UA & $\begin{array}{c}0.0390^{* * *} \\
(0.001)\end{array}$ & $\begin{array}{c}0.0288^{* * *} \\
(0.006)\end{array}$ & $\begin{array}{c}0.0331^{* * *} \\
(0.000)\end{array}$ \\
\hline $\begin{array}{l}\text { the explained variable of } \\
\text { lag phase }\end{array}$ & $\begin{array}{l}-0.0201 \\
(0.305)\end{array}$ & & \\
\hline control variable & control & control & control \\
\hline _cons & $\begin{array}{c}0.4012^{* * *} \\
(0.005)\end{array}$ & $\begin{array}{c}0.3840^{* * *} \\
(0.003)\end{array}$ & $\begin{array}{c}0.2456^{* * *} \\
(0.002)\end{array}$ \\
\hline region-fixed effect & YES & $\mathrm{NO}$ & YES \\
\hline time-fixed effect & YES & NO & YES \\
\hline Obs. & 1819 & 1819 & 1819 \\
\hline R-square & 0.2957 & 0.1920 & 0.2911 \\
\hline
\end{tabular}

Note: ${ }^{*}, * *$, and ${ }^{* * *}$ represent significance at $10 \%, 5 \%$ and $1 \%$ statistical level respectively; $p$ value is in parentheses.

\subsection{Further Analysis: The Moderating Role of City Level and City Size}

In the process of urban agglomeration, due to differences in the original resource endowment, existing economic foundation, policy development environment, and element attractiveness, the ULGUE promotion effect of different urban agglomerations is heterogeneous. Theoretically speaking, factors such as labor, capital, and technology tend to flow to cities with high administrative levels and large-scale cities. Governments at all levels also provide such cities with a good policy environment and financial support [36], so their ULGUE is improved relatively fast, but it is worth re-examining whether the ULGUE improvement effect of cities with agglomeration of different levels and scales is also in line with the law of diminishing marginal utility. That is, whether the cities with relatively poor resource conditions improve the ULGUE faster than the cities with better resource conditions in the process of agglomeration. To this end, the following two models are constructed to further verify the moderating effect of city level and city size.

$$
\begin{aligned}
& \operatorname{ULGUE}_{i t}=\beta_{0}+\beta_{2} U A_{i t} * \text { Level }_{i}+\gamma X_{i t}+v_{i}+\mu_{t}+\varepsilon_{i t} \\
& U L G U E_{i t}=\beta_{0}+\beta_{3} U A_{i t} * \text { Scale }_{i}+\gamma X_{i t}+v_{i}+\mu_{t}+\varepsilon_{i t}
\end{aligned}
$$

In Model (4), Level represents the city level. Taking into account that the 27 cities in this paper belong to the economic coordination association of central cities along the Yangtze River, this paper divides the city level into provincial capital cities (including Shanghai and Chongqing), regional central cities of Yangtze River coastal economic coordination association (except for the provincial capital cities), and other ordinary prefecture-level cities, and respectively examines the adjustment effect of city levels. $\beta_{2}$ represents the interaction of different levels of urban agglomeration on ULGUE, and the interpretation of other variables is the same as Model (3). The relevant results are shown in Table 5. Under the two-way fixed-effects model, the interaction coefficients of provincial capital cities are positive but not significant, and the interaction coefficients of regional central cities and ordinary prefecture-level cities are significantly positive at the levels of $5 \%$ and $1 \%$, respectively. The moderating effects of the city level from large to small are ordinary prefecture-level cities (0.0299), regional central cities (0.0284), and provincial capital cities (0.0107), indicating that the agglomeration between ordinary prefecture-level cities has the most significant effect on ULGUE improvement and regional central cities are second, while urban agglomerations in provincial capital cities do not significantly change the ULGUE level. It is further confirmed that urban agglomeration has a significant effect on the economic development quality of prefecture-level cities, and, at the same time, it also reflects that the city level presents a feature of diminishing marginal utility in the promotion effect of urban agglomeration on ULGUE. 
Table 5. Impacts of the urban agglomeration of different city levels on ULGUE.

\begin{tabular}{|c|c|c|c|c|c|c|}
\hline Variable & \multicolumn{2}{|c|}{$\begin{array}{c}\text { Provincial Capital } \\
\text { Cities }\end{array}$} & \multicolumn{2}{|c|}{$\begin{array}{c}\text { Regional Center } \\
\text { Cities }\end{array}$} & \multicolumn{2}{|c|}{$\begin{array}{c}\text { Ordinary Prefecture-Level } \\
\text { Cities }\end{array}$} \\
\hline UA * provincial capital cities & $\begin{array}{l}0.0165 \\
(0.116)\end{array}$ & $\begin{array}{l}0.0107 \\
(0.211)\end{array}$ & & & & \\
\hline $\mathrm{UA} *$ regional center cities & & & $\begin{array}{l}0.0252 * \\
(0.055)\end{array}$ & $\begin{array}{l}0.0284 * \\
(0.052)\end{array}$ & & \\
\hline UA * ordinary prefecture-level cities & & & & & $\begin{array}{c}0.0246^{* * *} \\
(0.006)\end{array}$ & $\begin{array}{l}0.0299 * * * \\
(0.008)\end{array}$ \\
\hline GI & $\begin{array}{c}-0.0453^{* *} \\
(0.014)\end{array}$ & $\begin{array}{c}-0.1058^{* * *} \\
(0.009)\end{array}$ & $\begin{array}{c}-0.0449 \text { ** } \\
(0.010)\end{array}$ & $\begin{array}{c}-0.1045^{* * *} \\
(0.003)\end{array}$ & $\begin{array}{c}-0.0549 * * * \\
(0.001)\end{array}$ & $\begin{array}{l}-0.1043^{* * *} \\
(0.003)\end{array}$ \\
\hline INFR & $\begin{array}{l}0.0002 \\
(0.534)\end{array}$ & $\begin{array}{c}-0.0009 \\
(0.140)\end{array}$ & $\begin{array}{l}0.0004 \\
(0.456)\end{array}$ & $\begin{array}{c}-0.0009 \\
(0.231)\end{array}$ & $\begin{array}{l}0.0004 \\
(0.657)\end{array}$ & $\begin{array}{c}-0.0009 \\
(0.143)\end{array}$ \\
\hline IS & $\begin{array}{l}0.2306^{* *} \\
(0.061)\end{array}$ & $\begin{array}{l}0.1854^{* * *} \\
(0.009)\end{array}$ & $\begin{array}{l}0.2132 * * \\
(0.076)\end{array}$ & $\begin{array}{l}0.2030^{* * *} \\
(0.008)\end{array}$ & $\begin{array}{l}0.1673^{* *} \\
(0.030)\end{array}$ & $\begin{array}{l}0.2222^{* * *} \\
(0.006)\end{array}$ \\
\hline RS & $\begin{array}{c}0.1288 * \\
(0.061)\end{array}$ & $\begin{array}{c}0.0047^{*} \\
(0.053)\end{array}$ & $\begin{array}{l}0.1440 * \\
(0.092)\end{array}$ & $\begin{array}{l}0.0060 * \\
(0.077)\end{array}$ & $\begin{array}{l}0.1627^{*} \\
(0.090)\end{array}$ & $\begin{array}{l}0.0067^{*} \\
(0.073)\end{array}$ \\
\hline TI & $\begin{array}{l}0.1049^{* * * *} \\
(0.000)\end{array}$ & $\begin{array}{c}0.1497^{* * *} \\
(0.000)\end{array}$ & $\begin{array}{l}0.1070^{* * *} \\
(0.001)\end{array}$ & $\begin{array}{c}0.1518^{* * *} \\
(0.000)\end{array}$ & $\begin{array}{c}0.1142^{* * * *} \\
(0.002)\end{array}$ & $\begin{array}{l}0.1458^{* * *} \\
(0.000)\end{array}$ \\
\hline region-fixed effect & $\mathrm{NO}$ & YES & $\mathrm{NO}$ & YES & $\mathrm{NO}$ & YES \\
\hline time-fixed effect & NO & YES & $\mathrm{NO}$ & YES & $\mathrm{NO}$ & YES \\
\hline _cons & $\begin{array}{l}0.4903^{* * *} \\
(0.000)\end{array}$ & $\begin{array}{c}0.4793^{* * *} \\
(0.000)\end{array}$ & $\begin{array}{l}0.4892^{* * *} \\
(0.000)\end{array}$ & $\begin{array}{l}0.4792 * * * \\
(0.000)\end{array}$ & $\begin{array}{l}0.4901 * * * \\
(0.000)\end{array}$ & $\begin{array}{l}0.4778^{* * *} \\
(0.000)\end{array}$ \\
\hline Obs. & 1819 & 1819 & 1819 & 1819 & 1819 & 1819 \\
\hline R-square & 0.0017 & 0.1922 & 0.0051 & 0.1922 & 0.0050 & 0.1922 \\
\hline
\end{tabular}

Note: ${ }^{*}, * *$ and ${ }^{* * *}$ represent significance at $10 \%, 5 \%$, and $1 \%$ statistical level respectively; $p$ value is in parentheses.

In Model (5), Scale is the city scale expressed by the number of urban populations at the end of the year. Since the 107 cities in the Yangtze River Economic Zone have an urban population of more than 500,000 at the end of the year between 2003 and 2019, according to the Notice on Adjusting the Criteria for Urban Scale Classification issued by the state council, they are divided into super large and megacities, big cities, and medium cities to examine the adjustment effect of urban scale. $\beta_{3}$ represents the interactive effects of urban agglomerations with different scales on ULGUE. The regression results are shown in Table 6: the interaction coefficients of super large and megacities are significantly positive under random effects, but not significant under fixed effects; the interaction coefficients of big cities and medium cities are both significantly positive; the moderating effects of city size from large to small are medium cities (0.0526), big cities (0.0386), and super large and megacities (0.0092). It further shows that urban agglomeration is more conducive to improving the land green use efficiency of relatively small cities, indicating that the adjustment effect of urban scale on urban agglomeration to promote ULGUE is also in line with the law of diminishing marginal utility.

Provincial capital cities and super large and megacities have generally developed into regional economic growth poles with more optimized factor allocation, more efficient technological advancement, and better infrastructure; therefore, the improvement of ULGUE by urban agglomeration may be relatively slow. In contrast, cities with relatively weak primitive resource conditions rely on agglomeration development models to accelerate the frequency of factor matching, the industrial division of labor, and knowledge diffusion, and it is easier to form a complementary symbiotic relationship, and the benefits of agglomeration are more pronounced. In addition, the diminishing marginal utility of the adjustment effect of city-level and city size also means that urban agglomeration not only helps to improve the ULGUE level, but a reasonable agglomeration area layout helps narrow the regional ULGUE gap to a certain extent. 
Table 6. Impacts of the urban agglomeration of different city sizes on ULGUE.

\begin{tabular}{|c|c|c|c|c|c|c|}
\hline Variable & \multicolumn{2}{|c|}{$\begin{array}{l}\text { Super Large and } \\
\text { Megacities }\end{array}$} & \multicolumn{2}{|c|}{ Big Cities } & \multicolumn{2}{|c|}{ Medium Cities } \\
\hline UA * super large and megacities & $\begin{array}{l}0.0206 * \\
(0.051)\end{array}$ & $\begin{array}{l}0.0092 \\
(0.534)\end{array}$ & & & & \\
\hline $\mathrm{UA} *$ big cities & & & $\begin{array}{c}0.0278 \text { ** } \\
(0.027)\end{array}$ & $\begin{array}{c}0.0386^{* * *} \\
(0.005)\end{array}$ & & \\
\hline $\mathrm{UA} *$ medium cities & & & & & $\begin{array}{l}0.0141 \\
(0.213)\end{array}$ & $\begin{array}{c}0.0526^{* * *} \\
(0.003)\end{array}$ \\
\hline GI & $\begin{array}{c}-0.0533 * \\
(0.087)\end{array}$ & $\begin{array}{c}-0.1065^{* *} \\
(0.012)\end{array}$ & $\begin{array}{c}-0.0482 * \\
(0.054)\end{array}$ & $\begin{array}{c}-0.0995^{* *} \\
(0.038)\end{array}$ & $\begin{array}{c}-0.0439 * * \\
(0.022)\end{array}$ & $\begin{array}{c}-0.1050 \text { ** } \\
(0.013)\end{array}$ \\
\hline INFR & $\begin{array}{l}0.0000 \\
(0.487)\end{array}$ & $\begin{array}{c}-0.0009 \\
(0.463)\end{array}$ & $\begin{array}{l}0.0001 \\
(0.821)\end{array}$ & $\begin{array}{c}-0.0010 \\
(0.890)\end{array}$ & $\begin{array}{l}0.0005 \\
(0.211)\end{array}$ & $\begin{array}{c}-0.0011 \\
(0.104)\end{array}$ \\
\hline IS & $\begin{array}{c}0.2488^{* *} \\
(0.086)\end{array}$ & $\begin{array}{c}0.1892^{* * *} \\
(0.004)\end{array}$ & $\begin{array}{c}0.2433^{* *} \\
(0.080)\end{array}$ & $\begin{array}{c}0.2034^{* * *} \\
(0.004)\end{array}$ & $\begin{array}{c}0.1376^{* *} \\
(0.061)\end{array}$ & $\begin{array}{c}0.1054^{* * *} \\
(0.004)\end{array}$ \\
\hline RS & $\begin{array}{c}0.1274 \text { * } \\
(0.098)\end{array}$ & $\begin{array}{l}0.0049 * \\
(0.066)\end{array}$ & $\begin{array}{l}0.1250 * \\
(0.094)\end{array}$ & $\begin{array}{c}0.0063 \text { * } \\
(0.075)\end{array}$ & $\begin{array}{l}0.2043^{*} \\
(0.090)\end{array}$ & $\begin{array}{c}0.0056^{*} \\
(0.070)\end{array}$ \\
\hline TI & $\begin{array}{c}0.1174 * * * \\
(0.001)\end{array}$ & $\begin{array}{c}0.1499 * * * \\
(0.000)\end{array}$ & $\begin{array}{c}0.1071 \text { *** } \\
(0.001)\end{array}$ & $\begin{array}{c}0.1525 * * * \\
(0.000)\end{array}$ & $\begin{array}{c}0.1011 \text { *** } \\
(0.002)\end{array}$ & $\begin{array}{c}0.1439^{* * *} \\
(0.000)\end{array}$ \\
\hline region-fixed effect & NO & YES & NO & YES & NO & YES \\
\hline time-fixed effect & $\mathrm{NO}$ & YES & NO & YES & $\mathrm{NO}$ & YES \\
\hline _cons & $\begin{array}{c}0.4923 * * * \\
(0.000)\end{array}$ & $\begin{array}{c}0.4792 * * * \\
(0.000)\end{array}$ & $\begin{array}{c}0.4886^{* * *} \\
(0.000)\end{array}$ & $\begin{array}{c}0.4788^{* * *} \\
(0.000)\end{array}$ & $\begin{array}{c}0.4888^{* * *} \\
(0.000)\end{array}$ & $\begin{array}{c}0.4782^{* * *} \\
(0.000)\end{array}$ \\
\hline Obs. & 1819 & 1819 & 1819 & 1819 & 1819 & 1819 \\
\hline R-square & 0.0015 & 0.1844 & 0.0094 & 0.1902 & 0.0023 & 0.1846 \\
\hline
\end{tabular}

Note: ${ }^{*}, * *$ and ${ }^{* * *}$ represent significance at $10 \%, 5 \%$, and $1 \%$ statistical level respectively; $p$ value is in parentheses.

\section{Conclusions and Discussions}

\subsection{Main Conclusions}

In the process of further promoting high-quality development, it is particularly important to pay attention to the interaction mechanism and influence effect between urban agglomeration and land-use efficiency, especially urban land green efficiency. However, the traditional development mode favoring economic aggregate growth makes problems such as population crowding, industrial isomorphism, environmental pollution, and resource shortage increasingly prominent. It is still unknown whether urban agglomeration will improve or reduce land-use efficiency. Therefore, this paper theoretically constructed the logical framework of the impact of urban agglomeration on urban land green use efficiency (ULGUE) and tested the impact of urban agglomeration on ULGUE based on the measurement of urban land green use efficiency (ULGUE) in 107 prefecture-level and above cities in the Yangtze River Economic Zone.

Compared with the existing research, the possible innovation of this paper lies in the theoretical framework of urban agglomeration affecting the urban land green use efficiency and use the differences-in-differences method to verify the promotion effect of urban agglomeration on ULGUE. The research content and research methods are a useful supplement to the existing "Urban Agglomeration-ULGUE" research paradigm.

The analysis results show that (1) urban agglomeration can affect ULGUE through polarization effect, trickle-down effect, and restraint effect. From 2003 to 2019, urban agglomeration has a promoting effect on ULGUE, and this research conclusion is robust. (2) The promotion effect of urban agglomeration on ULGUE has regional differences, showing a basic pattern of "ordinary prefecture-level cities $>$ regional central cities > provincial capital cities" and "medium cities $>$ large cities $>$ super and megacities".

\subsection{Discussions}

Regarding the research idea, compared with the existing research results [1-4], this paper breaks through the urban agglomeration of land-use performance assessment framework, introduces the concept of low-carbon city green land-use efficiency of study, reveals 
the mechanism of urban agglomeration on the urban land-use efficiency, extrapolated from theoretical logic out of the city agglomeration influencing the efficiency of the urban land use empirically, and avoids the dilemma of regional land use policy being overhead.

In terms of research results, the current research results mostly discuss the spatial distribution pattern and regional differences of urban agglomeration on urban land-use efficiency $[11-14,16]$, but do not systematically pay attention to the mediating effect of city grade and city size. Therefore, this paper further investigated the moderating effects of cities of different levels and sizes on ULGUE promotion, under the background of urban agglomeration development, in order to provide theoretical and empirical support for the exploration of a regional ULGUE differentiation promotion path.

\subsection{Policy Implications}

Based on the above conclusions, the following policy recommendations and enlightenments can be drawn: First, promote the depth and breadth of the integration of urban agglomeration in the Yangtze River Economic Zone as a whole, and further enrich the agglomeration development forms such as urban agglomerations and regional integration to give full play to the promotion effect of urban agglomeration on ULGUE. On the one hand, play the role of the market mechanism, promote the free flow and combination disposition of various elements between cities by optimizing the relationship between supply and demand, rely on the price mechanism to encourage the formation of a symbiotic relationship of complementary functions and regional division of labor between cities in the transformation and upgrading of industries, and realize the survival of the fittest in homogeneous industries and the replacement of high-polluting, low-efficiency enterprises with the help of competition mechanisms. On the other hand, local governments should break down the barriers of interest consolidation, strengthen intercity spatial linkages and exchanges and cooperation, adopt diversified policy tools to create conditions for factor flow, industrial transfer, technological innovation, and so on, and guide the central city to strengthen its radiating and leading role in peripheral cities. In addition, it is necessary to pay special attention to the leading role of scientific and technological innovation in urban agglomeration driving the efficient use of urban land, strengthen knowledge spillover, information sharing, human capital accumulation, stimulate the development of land use in the agglomeration area to the direction of high efficiency and intensification, and realize the sustainable economic, social, and ecological development of the city.

Second, based on the development needs of different types of cities, targeted development strategies for agglomeration should be formulated. The fact that urban agglomeration has a more significant effect on improving the ULGUE of cities with relatively poor resource bases shows that urban agglomeration cannot blindly expand and develop disorderly. On the one hand, provincial capital cities and super large and megacities can take measures, such as resource allocation and industrial transfer, to appropriately control the size of the urban population; on the other hand, the transformation of development mode, adjustment of economic structure, and optimization of public services should be adopted to promote the improvement of ULGUE. At the same time, ordinary prefecture-level cities and small and medium-sized cities should formulate fiscal and tax concessions, industrial support, and population settlement policies, based on their geographic location and existing industrial foundation, to attract the inflow of labor, industry, capital, and other factors. Thereby, while improving the ULGUE of such cities, it also narrows the ULGUE gap between cities.

The land is the space carrier for the exchange and interaction of all elements of the city. It systematically reveals the influence mechanism of urban agglomeration on ULGUE, which is the prerequisite for optimizing the urban development path and promoting highquality social and economic development. This paper focuses on the overall perspective of agglomeration development to examine its role in improving ULGUE, but urban agglomeration involves multiple dimensions such as population, industry, and capital. This paper did not measure the degree of urban agglomeration and its mechanism on ULGUE from a multidimensional perspective, nor did it deeply analyze the reverse effect of ULGUE on 
urban agglomeration and their coupling relationship, which are important directions for subsequent research.

Author Contributions: Conceptualization, methodology, and writing—original draft and editing, K.G.; funding acquisition, K.G., S.K. and D.C.; project administration and supervision, S.K. and D.C.; data curation, resources, software, and visualization, S.Z.; writing-review and editing, S.K. and S.Z. All authors have read and agreed to the published version of the manuscript.

Funding: This research was funded by "Jiangxi Provincial Association of Social Sciences" (grant number: 20GL37), "Education Department of Jiangxi Province" (grant number: GJJ191710), "Ministry of Education of the People's Republic of China" (grant number: 21YJC790056), and "Ministry of Education of the People's Republic of China" (grant number: 21YJC790006).

Institutional Review Board Statement: Not applicable.

Informed Consent Statement: Not applicable.

Data Availability Statement: The data presented in this study are available on request from the first author.

Conflicts of Interest: The authors declare no conflict of interest.

\section{References}

1. Wu, F.; Liu, Z. Research on the mechanism of how city group drive economic growth-Empirical evidences from 16 cities of Yangtze River Delt. Econ. Res. J. 2008, 43, 126-136.

2. Liu, T. The dilemma and solution of the coordinated development of Beijing-Tianjin-Hebei region. China Bus. Mark. 2015, 29, 83-88.

3. Luo, H.; Long, X.; Zhu, Y. A compound-administration-based research on the cooperation model of the " $3+5$ " urban agglomeration. Econ. Geogr. 2011, 31, 947-953.

4. Wang, D. Comprehensive measurement and evolution law of urbanization development quality of urban agglomeration in China. Chin. J. Popul. Sci. 2018, 1, 46-59.

5. Zhou, S.; Zhu, W. Must industrial agglomeration be able to bring about economic efficiency: Economies of scale and crowding effect. Ind. Econ. Res. 2013, 3, 12-22.

6. Liu, S.; Ye, Y.; Xiao, W. Spatial-temporal differences and driving factors of land use efficiency of four urban agglomerations in eastern China. Urban Probl. 2020, 4, 14-20.

7. Zhang, Y.; Chen, J.; Gao, J.; Jiang, W. The impact mechanism of urban land use efficiency in the Yangtze River Delta from the perspective of economic transition. J. Nat. Resour. 2019, 34, 1157-1170.

8. Chen, D.; Lu, X.; Kuang, B. Dynamic evolution and spatial convergence of urban land use efficiency in the middle reaches of the Yangtze River. China Popul. Resour. Environ. 2018, 28, 106-114.

9. Chen, D.; Li, J.; Hu, B. Spatial correlation characteristics of urban agglomerations land use efficiency in the middle reaches of the Yangtze River. Urban Probl. 2018, 9, 55-64.

10. Lu, X.; Chen, D.; Kuang, B. Indicator system design and regional difference of urban land use efficiency under the background of regional integration: A case of urban agglomeration in the middle reaches of the Yangtze River. China Popul. Resour. Environ. 2018, 28, 102-110.

11. Yu, B.; Su, Y. Research on the impact of industrial structure adjustment on land use efficiency and spillover effect: An empirical analysis based on the PSDM model and PTR model. China Land Sci. 2020, 34, 57-66.

12. Fu, H.; Wang, Z. Relationship between the adjustment of regional industrial structure and the efficiency of land use-A survey based on the level of urbanization. China Land Sci. 2020, 34, 69-78, 107.

13. Yue, L.; Xue, D. Study on the impact of new-type urbanization on urban land use efficiency of in China. Inq. Econ. Issues 2020, 110-120.

14. Yang, Y.; Lang, Y. Impact of urbanization on land use efficiency and its location differences in inland areas of China regarding the opening reform. China Land Sci. 2011, 25, 19-26. [CrossRef]

15. Liao, J.; Han, F.; Zhang, W.; Xu, D. The positive analysis of the effect of urbanization on land use efficiency in Changsha, Zhuzhou and Xiangtan. China Popul. Resour. Environ. 2010, 20,30-36.

16. Zhang, W.; Zou, J.; Wu, Q. Effect of production factors on urban land use efficiency: Based on provincial data of different development stages. Resour. Sci. 2020, 42, 1416-1427. [CrossRef]

17. Wu, X.; Zhou, J. Public investment, land use efficiency and regional differences of urban growth: Based on urban panel data of 1999 to 2006. Financ. Trade Res. 2011, 22, 68-77.

18. Song, Y.; Yeung, G.; Zhu, D.; Xu, Y.; Zhao, J. Spatio-temporal patterns and driving factors of urban land use efficiency at county level in Beijing-Tianjin-Hebei urban agglomeration. China Land Sci. 2021, 35, 69-78. 
19. Li, C.; Miao, M. Urban land use efficiency measurement of city group in middle reaches of the Yangtze River: Reality mechanism and spatiotemporal diversities. China Popul. Resour. Environ. 2017, 27, 157-164.

20. Nie, L.; Guo, Z.; Peng, C. Construction land utilization efficiency based on SBM-Undesirable and meta-frontier model. Resour. Sci. 2017, 39, 836-845.

21. Yue, L.; Li, W. Typical urban land use efficiency in China under environmental constraints based on DDF-Global MalmquistLuenberger index medeling. Resour. Sci. 2017, 39, 597-607.

22. Yang, Q.; Duan, X.; Ye, L.; Zhang, W. Efficiency evaluation of city land utilization in the Yangtze River Delta using an sbmundesirable model. Resour. Sci. 2014, 36, 712-721.

23. Verburg, P.H.; van Berkel, D.B.; Doorn, A.M.; van Eupen, M.; van den Heiligenberg, H.A.R.M. Trajectories of land use change in Europe: A model-base exploration of rural futures. Landsc. Ecol. 2010, 25, 217-232. [CrossRef]

24. Cai, S.; Ni, P. Polarization or trickle: The impact of urban scale on agricultural modernization. Economist 2017, 7, 46-55.

25. Qi, Y.; Zhao, Y.; Wang, M. Beyond the micro-mechanism of urban agglomeration economies: From the division of labor to division of knowledge. China Ind. Econ. 2012, 1, 36-45.

26. Martin, P. Growth and agglomeration. Int. Econ. Rev. 2010, 4, 947-968. [CrossRef]

27. Wei, H. Polarization trend and optimization of size distribution in China's urbanization process. China Ind. Econ. 2014, 312, 18-30.

28. Zhao, Y.; Wei, H. The government intervention, the division of labor of the function of the space of the city group, and the difference in district: On the effectiveness of regional policy in china. Manag. World 2015, 8, 14-29, 187.

29. Wei, P.; Yu, Y. The influence of urban industrial structure changes on economic efficiency: A case based on 285 cities of China. Urban Probl. 2018, 11, 4-11.

30. Zhu, Y.; Yang, L.; Lu, H.; Shen, X. Research on resource shortage, environmental damage and the effect of industrial agglomeration-Based on the empirical analysis of my country's provincial industrial agglomeration in the 21st century. Manag. World 2012, 11, 28-44.

31. Zheng, X.; Wang, H.; Zhao, Y. Can "province directly manage counties" promote economic growth?-Double difference method. Manag. World 2011, 8, 34-44, 65.

32. Yan, X.; Cheng, C.; Xu, C.; Yi, G.; Huang, X. Research hotspots and prospects of Yangtze River Economic Zones: Based on quantitative analysis of knowledge map. Econ. Geogr. 2018, 38, 16-25.

33. Li, D.; Zhao, Y.; Li, L. Change of environmental efficiency and environmental productivity of coal cities: Based on panel data of 11 cities in Shanxi province. J. Nat. Resour. 2021, 36, 618-633.

34. Hu, B.; Li, J.; Kuang, B. Evolution characteristics and influencing factors of urban land use efficiency differences under the concept of green development. Econ. Geogr. 2018, 38, 183-189.

35. Xiang, Y.; Wang, S.; Deng, C. Spatial differentiation and driving factor of green development efficiency of chemical industry in the Yangtze River Economic Belt. Econ. Geogr. 2021, 41, 108-117.

36. Liu, R.; Zhao, R. Did the national high-tech zones promote regional economic development?-Based on verifying the differencedifferences method. Manag. World 2015, 31, 30-38.

37. Yuan, J.; Hou, Q.; Cheng, C. The curse effect of corporate political resources-Based on the investigation of political connection and corporate technological innovation. Manag. World 2015, 1, 139-155.

38. Liang, L.; Yong, Y.; Yuan, C. Measurement of urban land green use efficiency and its spatial differentiation characteristics: An empirical study based on 284 cities. China Land Sci. 2019, 33, 80-87.

39. Wang, D.; Pang, X. Research on green land-use efficiency of Beijing-Tianjin-Hebei urban agglomeration. China Popul. Resour. Environ. 2019, 29, 68-76.

40. Lu, X.; Yang, X.; Chen, Z. Measurement and temporal-spatial evolution characteristics of urban land green use efficiency in China. China Popul. Resour. Environ. 2020, 30, 83-91.

41. Ji, Z.; Zhang, P. Spatial difference and driving mechanisms of urban land use efficiency under environmental constraints: Based on 285 cities in China. China Land Sci. 2020, 34, 72-79.

42. Bai, Y.; Shi, L.; Dang, Y. Spatial agglomeration and diffusion of urban agglomerations in middle reaches of the Yangtze River: A flow test based on labor factors of 18 industries in 31 cities. Econ. Geogr. 2016, 36, 38-46. 